\title{
WestVirginiaUniversity
}

THE RESEARCH REPOSITORY @ WVU

Graduate Theses, Dissertations, and Problem Reports

2015

\section{Identification of a critical gene in the dihydroergot alkaloid pathway}

Yulia Bilovol

Follow this and additional works at: https://researchrepository.wvu.edu/etd

\section{Recommended Citation}

Bilovol, Yulia, "Identification of a critical gene in the dihydroergot alkaloid pathway" (2015). Graduate Theses, Dissertations, and Problem Reports. 5212.

https://researchrepository.wvu.edu/etd/5212

This Thesis is protected by copyright and/or related rights. It has been brought to you by the The Research Repository @ WVU with permission from the rights-holder(s). You are free to use this Thesis in any way that is permitted by the copyright and related rights legislation that applies to your use. For other uses you must obtain permission from the rights-holder(s) directly, unless additional rights are indicated by a Creative Commons license in the record and/ or on the work itself. This Thesis has been accepted for inclusion in WVU Graduate Theses, Dissertations, and Problem Reports collection by an authorized administrator of The Research Repository @ WVU. For more information, please contact researchrepository@mail.wvu.edu. 


\title{
Identification of a critical gene in the dihydroergot alkaloid pathway
}

\author{
Yulia Bilovol \\ Thesis submitted \\ to the Davis College of Agriculture, Natural Resources, and Design \\ at West Virginia University \\ in partial fulfillment of the requirements for the degree of \\ Master of Science in \\ Applied and Environmental Microbiology
}

Daniel G. Panaccione, Ph.D., Chair

Gary K. Bissonnette, Ph.D

Alan J. Sexstone, Ph.D

Division of Plant and Soil Sciences

Morgantown, West Virginia

2015

Keywords: Ergot alkaloids, Aspergillus, mycotoxins, fungi

Copyright 2015 Yulia Bilovol 


\section{Abstract \\ Identification of a critical gene in the dihydroergot alkaloid pathway \\ Yulia Bilovol}

Ergot alkaloids, bioactive compounds produced by some species of fungi, have had significant impacts on agriculture and medicine. Claviceps purpurea and Epichlö̈ spp. are ergot alkaloid producers associated with agricultural commodities and negatively affect humans and grazing animals, respectively. Another ergot alkaloid-producing fungus called Aspergillus fumigatus is ubiquitous in the environment; infections arising after inhaling the spores may kill immunocompromised persons. Ergot alkaloids and their derivatives are utilized by the pharmaceutical industry for the development of drugs. Some of these drugs are derived from lysergic acid, whereas others are derived from dihydrolysergic acid. Lysergic acid derivatives have vasoconstrictive properties and are used as treatment of acute headaches and in obstetrics.

Dihydrolysergic acid derivatives are vasorelaxant and are the basis of drugs that help treat dementia, Alzheimer's, and Cushing's diseases. The ergot alkaloid pathway has largely been elucidated, but unknown steps remain, particularly in the dihydrolysergic acid branch. The gene encoding the enzyme that oxidizes festuclavine, a key intermediate in the dihydrolysergic acid branch, to fumigaclavine B is unknown. Based on DNA sequence analysis, we hypothesized that the $A$. fumigatus gene eas $M$ encodes this enzyme. To test this hypothesis we knocked out eas $M$ by inserting a hygromycin resistance gene in the middle of its coding sequence and transforming

A. fumigatus with this construct. HPLC analysis showed that knock out of eas $M$ blocked the ergot pathway at festuclavine, and three downstream products (fumigaclavines B, A, and C) were eliminated. PCR results showed the hygromycin resistance fragment was present in the middle of eas $M$-coding sequences in these transformants. Complementation, or reintroduction of the native non-disrupted eas $M$ gene restored the ability of the fungus to produce all downstream compounds. These results indicate that eas $M$ encodes the enzyme that oxidizes festuclavine to fumigaclavine B. The festuclavine-accumulating strain of $A$. fumigatus will be instrumental in future studies investigating the biosynthesis of dihydrolysergic acid derivatives, which are derived from festuclavine and are the basis for important vasorelaxant drugs. 


\section{Acknowledgments}

This thesis would not have been possible without the help of a multitude of individuals and resources. I would first like to thank the Division of Plant and Soil Sciences for their support and the USDA grant 2012-67013-19384 (to Dr. Panaccione) which has funded this research.

Most importantly, I would like to thank my advisor Daniel Panaccione for his guidance, patience, and support. Thank you for graciously accepting me into your lab, for helping me grow as a student, a scientist, and a person. I am deeply grateful for the opportunity to have learned from you during my time as a graduate student. I would also like to thank my committee members Dr. Bissonnette and Dr. Sexstone. Dr. Bissonnette sparked my interest in microbiology during my time as an undergraduate student in his general microbiology course. He has always been kind and has made me feel welcome in the department by offering words of encouragement and answering my questions. Dr. Sexstone introduced graduate school to me and has been a wealth of knowledge during my time in this department.

A big thank you to Katy Ryan, my lab mate for her help in and out of the laboratory. She has been and invaluable resource. Furthermore, I would like to thank the graduate students in the Division of Plant and Soil Sciences. Each and every one of them has made this journey interesting and fun. We have shared a lot of knowledge and a lot of laughs, you all will be sorely missed.

Last but not least, I want to express my appreciation to those closest to me. My family has been incredibly supportive throughout my life and especially in the last two years. Thank you for inspiring me to reach my dreams. To Lukas, thank you for your encouragement throughout my academic and personal journey, you are my compass. 


\section{Table of Contents}

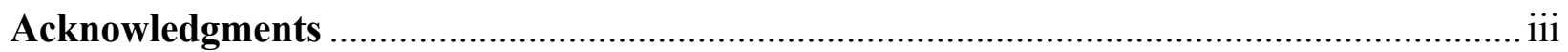

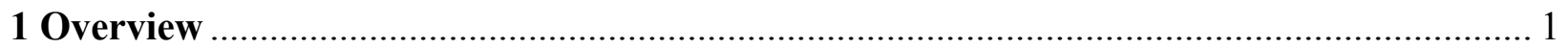

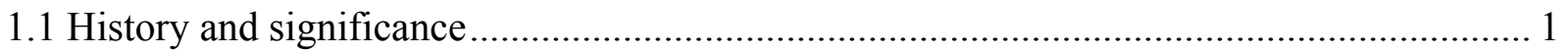

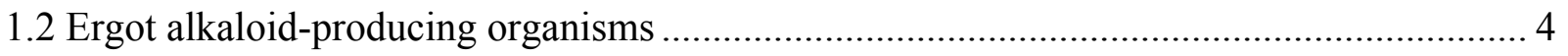

1.3 Ergot alkaloid cluster, pathway, and unknown steps ............................................. 6

1.4 Justification for choosing eas $M$ as a target gene .......................................................... 7

1.5 Benefits of research - agricultural and pharmaceutical implications .............................. 8

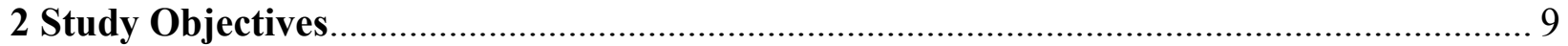

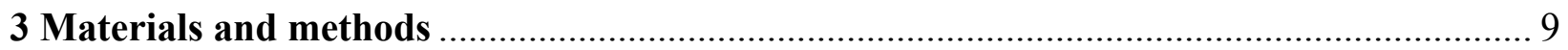

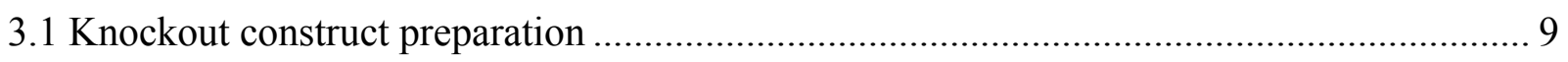

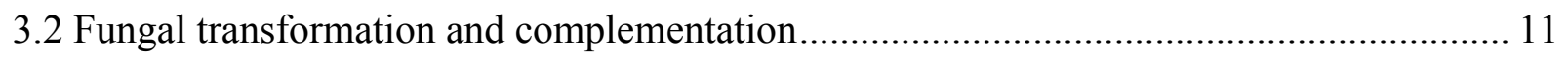

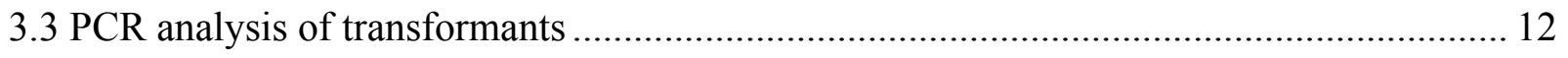

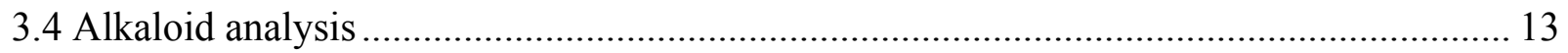

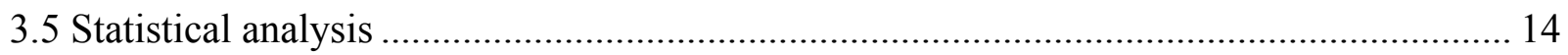

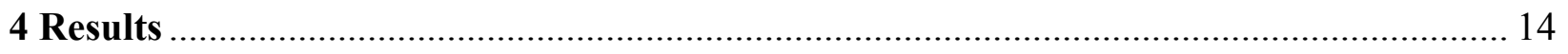

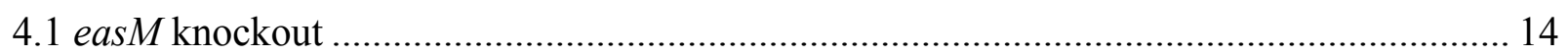

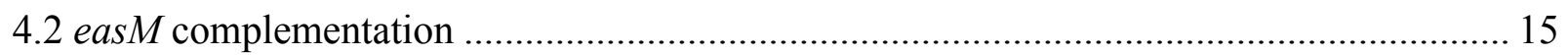

4.3 Quantitative analysis of ergot alkaloids ................................................................... 16

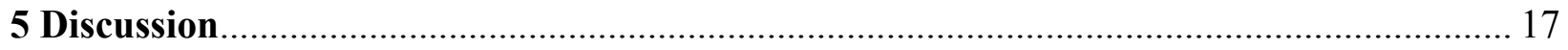

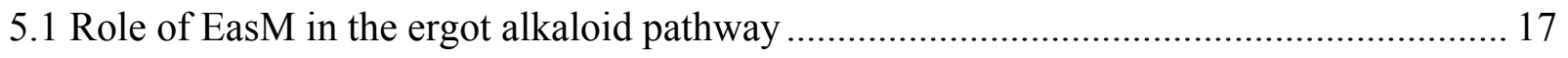

5.2 Differences in alkaloid quantity among wild type, knockout, and complemented strains.. 17

5.3 Additional studies and future directions................................................................. 19

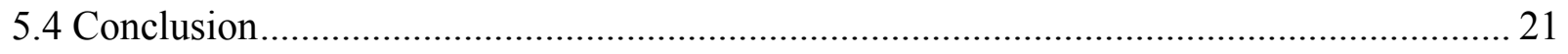

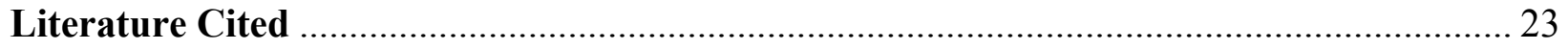

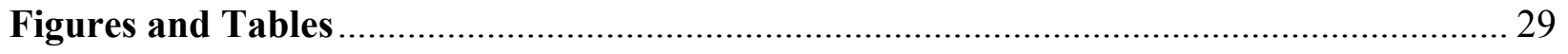

Figure 1: Divergence of the ergot alkaloid pathway ....................................................... 29

Figure 2: Ergot alkaloid synthesis gene cluster of A. fumigatus (A), Epichloë sp. Lp1 ergot

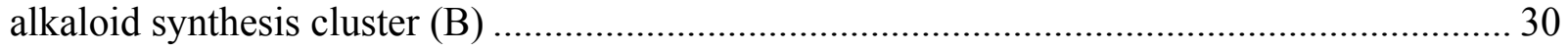

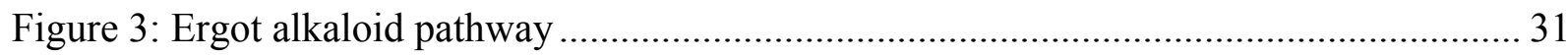

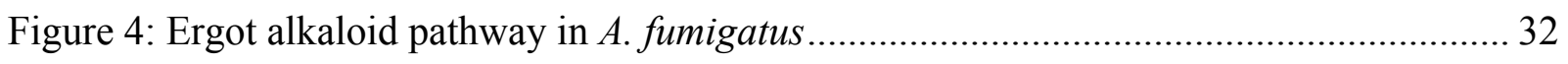

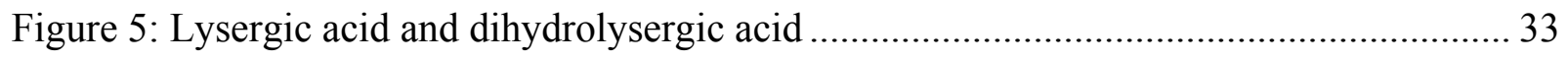


Figure 6: Schematic representation of eas $M$ locus (A), PCR products (B) ........................... 34

Figure 7: High performance liquid chromatography (HPLC) results .................................. 35

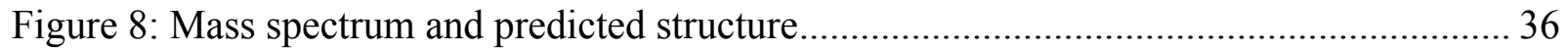

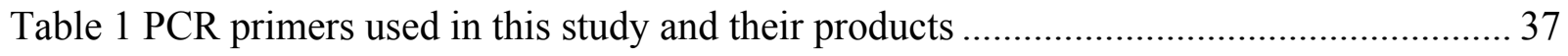

Table 2 Mean alkaloid concentrations .............................................................................. 38 


\section{Overview}

Ergot alkaloids are bioactive compounds that can be produced by several different species of fungi in the families Clavicipitaceae and Trichocomaceae. These fungi, collectively, can be found in a variety of environments. Some can live as saprotrophs, whereas others can infect a variety of hosts, including grasses and cereals, or humans and animals. The ergot alkaloid pathway for all ergot alkaloid-producing fungi appears to be shared in the early steps, but their divergence at the chanoclavine-I aldehyde branch point (Figure 1) leads to unique end products that are dependent upon the species of fungus producing them (Panaccione et al. 2012). Typical products of these pathways include clavines and lysergic acid derivatives. The synthesized chemicals are pertinent in medicine, as they are used in pharmaceuticals, and are of concern in agriculture due to their effect on grazing animals. Ergot alkaloid biosynthesis has been well studied but a few unknown steps remain thus, further understanding of these compounds can be of benefit for applications in medicine and agriculture in the future.

\subsection{History and significance}

Ergot alkaloid-producing fungi established their significance throughout history. Agricultural notoriety took root when grain crops, particularly rye, infected with Claviceps purpurea caused products made from the infected cereals to be unsafe to eat. A hard black structure called a sclerotium replaces seed within the flowering portion of the grass and was processed as grain until its deleterious effects were discovered. The mycotoxins produced within these hard overwintering structures are able to survive baking temperatures and digestive conditions, thus making them tenacious toxins (Merkel et al. 2012). Ergot poisoning or ergotism causes a variety of symptoms from hallucinations to gangrene of the extremities and convulsions 
(Belser-Ehrlich et al. 2013). "St. Anthony's fire” or ignis sacer derives its name from the burning sensations occurring in the extremities due to the vasoconstrictive activities of ergot alkaloids ingested from ergot-infected grains. Ergotism left such an impression on history that renaissance painters like Matthias Grünewald and Hieronymus Bosch immortalized the disease through art. Fortunately, the diversification of diets and processing or screening of grain has largely alleviated ergotism's threat to consumers. Belser-Ehrlich et al. (2013) chronicle the major outbreaks of ergotism in humans since 1900 occurring in Russia in 1926-1927, England in 1927, Ireland in 1929, France in 1951, India in 1956-1957 and 1975, and Ethiopia in 1977-1978 and 2001. Luckily these outbreaks have not been equal in magnitude to those of the Middle Ages, and they were confined to remote areas or countries where regulations are not rigorous. Current regulations in developed countries around the world limit the amount of ergot that can be consumed by humans; for example, in the US the limit for ergot presence in rye cannot exceed 0.3 percent (Code of Federal Regulations 2001) and is lower for other products like oats, wheat, and barley.

Deleterious effects from ergot-producing fungi may also be experienced by livestock ingesting contaminated grasses or feed. Epichloë species, grass symbionts capable of producing ergot alkaloids, have been known to cause grazing livestock to experience unfavorable symptoms including vasoconstriction at the extremities resulting in gangrene (Schardl et al. 2006). Epichlö endophytes are known to produce four classes of alkaloids: ergot alkaloids (lysergic acid and ergovaline), indolediterpenes, pyrrolopyrazine, and aminopyrrolizidines that act as insecticidal compounds. Among these four classes, ergot alkaloids and indolediterpenes can affect vertebrates (Schardl and Phillips 1997). Coupled with environmental conditions, ergot toxicosis has varying effects on livestock. For example in heat stress conditions, animals that 
ingest the toxins lose control of body temperature, water balance, experience necrosis of adipose tissue, and have the tendency to eat less food. Ingestion of toxins in cold conditions leads to gangrene. In general, toxin ingestion causes a reduction of lactation and fertility and, particularly in horses, miscarriages, fetal abnormalities, and stillbirths (Schardl and Phillips 1997). Economic losses due to livestock toxicosis may be ameliorated by using genetic techniques targeting toxin producing genes of mycobionts. Genetic engineering techniques are being explored, but since endophytes benefit their plant hosts, special attention needs to be paid to the acuteness of toxicosis in animals and environmental and genetic factors in selecting agronomically desirable endophytes that will maintain their endophyte niche but will not harm grazing livestock. Thus, there is still much to be explored regarding the control of toxin producing endophytes.

Ergot alkaloids' effects on history were not all negative; people have attempted to harness their biological activities for religious ceremonies and medicinal applications. In ancient Aztec cultures, priests would eat morning glory seeds with their associated alkaloid producing endophytes to induce psychoactive effects as a means of communication with the gods (Hofmann, Rätsch, \& Schultes 1992). Albert Hoffman was the research chemist responsible for the discovery of lysergic acid diethylamide (LSD) and also investigated the psychoactive effects of seed endophytes and hallucinogenic mushrooms eaten in South America (Hoffman 1980). His studies indicated that the chemicals lysergic acid amide, ergonovine, and lysergic acid hydroxyethylamide (the main active component in the preparations ingested by the indigenous people of South America) are closely chemically related to LSD. Beyond the psychoactive effects, lysergic acid derivatives act on smooth muscle tissues which is why midwives in the Middle Ages utilized ergot alkaloids to aid in child birth; however, because concentrations of alkaloid were difficult to control in preparations and often caused complications they eventually 
fell out of use (Tudzynski, Correia, \& Keller 2001). With time, more became known about alkaloid activities and with it the ability to control dosage. Alkaloids began being incorporated into headache medications and a host of other drugs on the market today.

\subsection{Ergot alkaloid-producing organisms}

Aspergillus fumigatus is a common saprophyte in the phylum Ascomycota. A. fumigatus plays a role in an agricultural aspect: it spoils stored crops, such as animal feed especially in warm, moist conditions (Scudamore \& Livesey 1997). A. fumigatus is ubiquitous due to its key role in environmental decay; thus, it can be found at especially high concentrations around composting facilities and other areas where there is a large amount of decomposing matter. $A$. fumigatus conidia are minute and very buoyant (Panaccione and Coyle 2005) allowing them to travel great distances, aiding the organism in spread and infection. The presence of A. fumigatus in air has been associated with respiratory ailments and allergic symptoms in humans. In immunocompromised persons it may manifest as invasive aspergillosis or systemic spread of infection, with a mortality rate of more than fifty percent. In healthy individuals, the immune system will typically clear the infection, but in some cases the fungus may colonize cavities within lung tissue or sinuses resulting in aspergilloma or fungus ball (Denning 1998).

A. fumigatus synthesizes alkaloids called clavines, or more particularly festuclavine, fumigaclavine $\mathrm{A}$, fumigaclavine $\mathrm{B}$, and fumigaclavine $\mathrm{C}$, with fumigaclavine $\mathrm{A}$ or fumigaclavine C typically occurring as the end product in the pathway (Robinson and Panaccione 2012). Alkaloid production is associated with asexual reproduction, with alkaloid metabolites localized in the conidia (Coyle et al. 2007). Aspergillus fumigatus is an ideal candidate for the study of ergot alkaloid biosynthesis because it grows quickly in the saprophytic state in vitro yielding 
measureable amounts of chemical. Its genome has also been sequenced, with numerous studies investigating genes within the ergot alkaloid gene cluster through knockout and complementation studies. Resulting knockout strains are important for serving as recipients for the expression of ergot alkaloid synthesizing genes of other fungi. This study follows suit with the knockout and complementation of a gene with an unassigned role in the ergot alkaloid gene cluster. Future studies will be able to utilize the knockout strain of A. fumigatus as a recipient for a gene of interest in an attempt to understand dihydroergot alkaloid biosynthesis.

Epichloë spp. (formally Neotyphodium spp.) are another genus of fungi in the family Clavicipitaceae that produce ergot alkaloids; they are grass symbionts that colonize above ground portions of plants, growing in the intercellular spaces. Tall fescue and perennial rye grass are two of the most commonly studied grasses relating to Epichloë spp. as symbionts. Symbiotic or endophytic relationships between grasses and Epichloë species are seed transmissible (Bacon $\&$ White 2000) meaning that the symbiotic fungus not only grows in the intercellular spaces of the plant but that it will grow into the developing seed for the purpose of dispersal and colonization when the new seed germinates. The mutualism provides the plant with drought tolerance, enhanced photosynthetic rate, and growth (Clay \& Schardl 2002). Ergot alkaloid production in plants has also been shown to deter herbivory; a study found that rabbits preferred to eat grasses colonized by endophytic fungi that were alkaloid free versus grasses containing alkaloids or even endophyte-free grasses (Panaccione et al. 2006). Additionally, it has been well documented that biologically active alkaloids of the fungus have insecticidal activities, conferring protection to its host and increasing its competitiveness (Schardl et al. 2004). The lysergic acid derivatives produced by Epichloë spp. cause livestock feeding on these grasses to experience detrimental effects such as poor fertility, convulsions, poor weight gain, gangrene, 
and even death (Schardl \& Phillips 1997). Studying alkaloid producing endophytes is worthwhile to understand how they can be better managed out in the field to decrease their effects on the economic loses pertaining to grazing animals.

\subsection{Ergot alkaloid cluster, pathway, and unknown steps}

Ergot alkaloid synthesizing genes are found in clusters; however, the clusters vary depending on the organism (Figure 2). Genetic variation within clusters between species allows for the rich diversity in the ergot alkaloid pathway allowing some species to produce lysergic acid derivatives, dihydrolysergic acid derivatives, ergopeptines, or fumigaclavines (Figures $1 \&$ 3). Arrangement of genes, variation in coding sequences for alleles, and even the presence or absence of some genes within clusters vary, and may be observed via sequencing of the ergot alkaloid clusters (Figure 2) (Schardl et al. 2013; Coyle and Panaccione 2005). For example, genes unique to the A. fumigatus cluster (easN, and easL,) are responsible for the production of different fumigaclavines (Figure 4). All of these genes have been sequenced, but some of their roles have not yet been assigned; this is the primary focus of this study.

Early steps in the ergot alkaloid synthesis pathway are evolutionarily conserved among alkaloid producing fungi (Figure 3). An early step in the ergot alkaloid pathway is the prenylation of tryptophan by DMATrp synthase, coded by the $d m a W$ gene. Genetic and biochemical analyses demonstrated that it is the determining step in the ergot pathway for $A$. fumigatus (Coyle and Panaccione 2005). Knockout of dmaW in A. fumigatus completely eliminated all ergot alkaloids from the mutant, and the complementation restored production. The next important feature of the pathway is the chanoclavine-I aldehyde intermediate, at which the pathway diverges and varying terminal branch products are made according to individual 
producers (Panaccione et al. 2012). Understanding which genes are responsible for each step in these pathways is critical for the study of the biosynthesis of the biologically important end products. Investigation of one of the remaining unknown steps, for which eas $M$ is hypothetically responsible, will help complete the pathway for the production of fumigaclavines in A. fumigatus and will be instrumental for future research of dihydroergot alkaloid biosynthesis.

\subsection{Justification for choosing eas $M$ as a target gene}

One of the few remaining unknown steps in fumigaclavine synthesis for A. fumigatus is the oxidative step responsible for modifying festuclavine into fumigaclavine B. Two candidate genes for this step are eas $M$ and eas $K$, which are located in the ergot alkaloid gene cluster (Figures $2 \& 4$ ) and for which roles have not yet been assigned. Two pieces of evidence used to choose easM as the candidate gene for this study were as follows. First, the proposed structure for the protein coded by eas $M$ indicates that it is a P450 monooxygenase (Panaccione 2010). This putative function aligns well with the activity required to convert festuclavine to fumigaclavine B, which differs only by the presence of a hydroxyl group. Second, comparative genomics of $A$. fumigatus with Penicillium commune, organisms with similar clusters and similar ergot alkaloid profiles, indicates that the presence of eas $M$ and not eas $K$ correlates with the ability to produce ergot alkaloids (Panaccione 2010; Coyle \& Panaccione 2005; Wallwey and Li 2011). Both eas $M$ and eas $K$ are present in $A$. fumigatus but $P$. commune only has eas $M$ while still retaining the ability to produce alkaloids (Wallwey and Li 2011). This leads us to hypothesize that eas $K$ is not essential for alkaloid production or that it may be redundant with eas $M$. Both of these clues suggest that eas $M$ is the responsible gene for festuclavine oxidation to fumigaclavine B. 


\subsection{Benefits of research - agricultural and pharmaceutical implications}

Pharmaceutically, derivatives of the ergot alkaloid pathway are important because they can be incorporated into drugs. Ergot alkaloids are similar in molecular structure to neurotransmitters that interact with serotonin, dopamine, adrenaline, and neurodreneline receptors (Belser-Ehrlich et al. 2013; Panaccione 2011). These similarities are attributed to the psychoactive and/or vasoconstrictive or relaxant properties associated with these mycotoxins. Mycotoxin agonism for serotonin-2A receptors is thought to be responsible for the hallucinogenic properties of ergot alkaloids (Vollenweider et al. 1998). Vasoconstrictive symptoms in the extremities are linked to alkaloids interacting with serotonin-1B/D receptors (Anderson et al. 1987). Vasoconstrictive properties of lysergic acid derivatives have been used to induce labor, terminate postpartum bleeding, and mimic dopamine (Hoffman 1980). Lysergic acid derivatives are currently ingredients in drugs that treat type II diabetes (Via et. al. 2010) and act as neurotransmitter agonists (Cabergoline) for treatment of pituitary disorders like Parkinson's disease. It is important to note that because alkaloids have affinities for many receptors and can act as agonist or antagonists, their biological activities are not simple and often associated with many side-effects (Panaccione 2010).

Dihydrolysergic acid derivatives differ from lysergic acid derivatives in that they lack a double bond in their D ring (Figure 5) and thus have vasorelaxant effects, opposite of those produced by lysergic acid derivatives. Dihydrolysergic acid derivatives are used in the treatment of disorders like dementia (Nicergoline) (Fioravanti \& Flicker 2001), Alzheimer's, and Cushing's disease (Bracco et al. 2004). Patients with frequent, moderate headaches may receive a prescription from their provider for a nasal spray or injection called Migranal ${ }^{\circledR}$ a 
dihydroergotamine (Valeant Pharmaceuticals, QC, Canada); tablets may also be available under the name Ergomar ${ }^{\circledR}$ and contain ergotamine (Rosedale Therapeutics, TN, USA) (Reddy 2013).

Thus, the production of dihydrolysergic acid by a novel fungus may have agricultural and pharmaceutical benefits. Dihydrolysergic acid-producing fungi in agriculture may counteract the vasoconstrictive effects of natural Epichloë-infected grasses. The development of a more efficient process for attaining dihydrolysergic acid, via biosynthesis rather than chemically, can be beneficial to the pharmaceutical industry by lowering costs.

\section{Study Objectives}

This study will elucidate an unknown step in fumigaclavine synthesis in A. fumigatus. I will test the hypothesis that the product of eas $M$ is necessary for oxidizing festuclavine to fumigaclavine B. One potentially significant outcome of this work would be the production of a strain of A. fumigatus that can be used for further studies on the biosynthesis of dihydrolysergic acid.

\section{Materials and methods}

\subsection{Knockout construct preparation}

The easM gene was PCR amplified from A. fumigatus template DNA in a reaction primed with eas $M$ forward and eas $M$ reverse primers (Table 1, Figure 6A). The EasM reaction consisted of $25 \mu \mathrm{L}$ mixture containing: 1 X GoTaq Flexi buffer (Promega, Madison, WI), 1.5 $\mathrm{mM} \mathrm{MgCl} 2,200 \mu \mathrm{M}$ each deoxynucleotide triphosphate, $1 \mu \mathrm{M}$ of each designated primer, and 2.5 units GoTaq Flexi DNA polymerase (Promega, Madison, WI). The thermocycler program that carried out the procedure began with a denaturation step at $95^{\circ} \mathrm{C}$ for 3 minutes, then 35 
cycles of: $95^{\circ} \mathrm{C}$ for 30 seconds, $55^{\circ} \mathrm{C}$ for 30 seconds, and $72^{\circ} \mathrm{C}$ for 3 minutes, and ending with a final extension of $72^{\circ} \mathrm{C}$ for 5 minutes. The eas $M$ fragment is 3,067 bases long, the length of which was confirmed by gel electrophoresis (Figure 6). The eas $M$ fragment was then ligated into plasmid pCR 2.1 to obtain a more concentrated product, and the ligation was transformed into Escherichia coli. E. coli colonies were grown on ampicillin-containing medium to select plasmid containing colonies. The presence of eas $M$ in the plasmid was determined by PCR primed with eas $M$ forward and eas $M$ reverse primers, in the same PCR program as described above. The product was then cleaned using a QIAquick column (Qiagen, Gaithersburg, MD).

To disrupt the eas $M$ coding sequence in the plasmid, it was digested with restriction enzymes SmaI and ClaI which cut out 1,091 bases located in the middle of the 3,067 base pair sequence of eas $M$. The 1,443 base pair hygromycin resistance gene was amplified from template pCB1004 in a PCR reaction primed with SmaHygF and ClaHygR using the same thermocycler program as described above; recognition sites for SmaI and ClaI were included in the 5' ends of these primers and were used to introduce the hygromycin-resistance fragment into the gap of the eas $M$ plasmid by ligating the SmaI-ClaI cut hygromycin resistance fragment with the SmaI-ClaI cut eas $M$-pCR 2.1 plasmid. The ligation was then transformed into $E$. coli and the resulting colonies were screened for the correct fragment via PCR using eas $M$ forward and reverse primers to visualize the $3,417 \mathrm{bp}$ fragment of disrupted eas $M$ with the hygromicin fragment in the middle (Figure 6). As an additional check the fragment was digested again with restriction enzymes SmaI, ClaI, EcoRI to visualize that the cut portions corresponded to the predicted sizes. The insert of the final plasmid sample was amplified by PCR using eas $M$ forward and eas $M$ reverse primers and Phire Hot Start II polymerase (Thermo-Fisher Scientific, Waltham, MA) in a program starting with a $98^{\circ} \mathrm{C}$ denaturation for 30 seconds, followed by 35 cycles of: $98^{\circ} \mathrm{C}$ for 15 
seconds, $65^{\circ} \mathrm{C}$ for 15 seconds, with a final extension at $72^{\circ} \mathrm{C}$ for 1 minute. The product was then cleaned using QIAquick column (Qiagen, Gaithersburg, MD).

\subsection{Fungal transformation and complementation}

The eas $M$-HYG fragment was transformed into $A$. fumigatus by established methods (Coyle and Panaccione 2005) and grown on hygromycin selective medium. Only cells that took up the eas $M$-hygromycin knockout construct were able to grow since they had the hygromycin resistance gene (Figure 6A).

To start the culture for transformation, A. fumigatus strain Af293 was grown on malt extract agar for approximately three days. Then, $10 \mathrm{~mL}$ of malt extract broth was used to create a spore suspension which was subsequently inoculated into an additional $20 \mathrm{~mL}$ of malt extract broth in a $50 \mathrm{~mL}$ flask and incubated overnight (approximately 16 hours) on an orbital shaker at $37^{\circ} \mathrm{C}$ and $80 \mathrm{rpm}$. Mycelium was pelleted in a $30-\mathrm{mL}$ Nalgene Oak Ridge tube. Protoplasts were prepared by suspending the mycelial pellet in $15 \mathrm{~mL}$ of $0.7 \mathrm{M} \mathrm{NaCl}, 40 \mathrm{mg}$ lysing enzyme (Sigma-Aldrich, Saint Louis, MO), and 1 g VinoTaste Pro (Novozymes, Switzerland) and allowing the mixture to incubate at $30^{\circ} \mathrm{C}$ for two hours. Protoplasts were separated from debris via filtration through a Miracloth (Calbiochem, San Diego, CA) funnel. Protoplasts were then washed by successively centrifuging (3,000 rpm for $5 \mathrm{~min}$ ) and resuspending in the following solutions: $10 \mathrm{~mL} 0.7 \mathrm{M} \mathrm{NaCl}, 5 \mathrm{~mL} \mathrm{STC}$ (1M sorbitol, $50 \mathrm{mM}$ tris-Cl pH 7.4, $50 \mathrm{mM} \mathrm{CaCl}$ ), and finally several hundred $\mathrm{uL}$ of STC to achieve protoplast concentration of $5 \times 10^{6}$ protoplasts per $100 \mathrm{uL}$. A solution of two parts $60 \%$ polyethylene glycol (PEG) and one part PEG amendments (1.8 M KCl, $150 \mathrm{mM} \mathrm{CaCl}_{2}, 150 \mathrm{mM}$ Tris $\left.\mathrm{pH} 7.4\right)$ was then added to comprise $1 / 5$ of the volume. Aliquots of $125 \mu \mathrm{L}$ of protoplast-PEG solution were added to $1.5 \mathrm{~mL}$ sterile 
tubes, along with $10 \mu \mathrm{L}$ of the eas $M$ knockout construct and then incubated on ice for 30 minutes. An additional $1 \mathrm{~mL}$ of PEG solution was added, the mixture was incubated at room temperature for 20 minutes and then plated onto Hygromycin (InvivoGen, San Diego, CA) selective medium.

A complementation assay was carried out to demonstrate that the phenotype of the eas $M$ knockout was due to the disruption at eas $M$ and not some other unrelated mutation in the colony. The wild-type allele of eas $M$ was first amplified from eas $M$ forward and eas $M$ reverse primers with Phire polymerase. The PCR product was cleaned up using Qiagen (Qiagen, Gaithersburg, MD) clean up kit. Next, a transformation was performed, as described above with Phleomycin (InvivoGen, San Diego, CA) resistance conferred by pBCphelo as the selectable marker onto Phleomycin (InvivoGen, San Diego, CA) selective medium.

\subsection{PCR analysis of transformants}

DNA preparations from each strain (wild type, eas $M$ knockout, and eas $M$ complemented) were checked via three PCR assays (Figure 6B). A wild-type screen primed with the easM forward and eas $M$ reverse primers checked for the size of the allele at the eas $M$ locus. A three prime screen with easNtowardM with YG primers checked for presence of the 3' portion of eas $M$ adjacent to the hygromycin resistance gene. A five prime screen based on easDinF with HY primers checked for the presence of the 5' portion of eas $M$ adjacent to the hygromycin resistance gene. 


\subsection{Alkaloid analysis}

To investigate the types of ergot alkaloids that accumulated in transformants, samples were analyzed by high performance liquid chromatography (HPLC) with fluorescence detection and liquid chromatography mass spectrometry (LC/MS).

Six replicate plates of A. fumigatus Af293 wild type, easM knockout, and easM complemented were grown on malt extract agar for two weeks. Cultures were extracted by removing a $\sim 50 \mathrm{~mm}^{2}$ sample area via the wide end of $1,000-\mu \mathrm{L}$ pipette tip and suspending it in HPLC-grade methanol for an hour, rotating on a carousel. After centrifugation, supernatant (20 $\mu \mathrm{L}$ ) of the extraction was analyzed via reverse phase HPLC. Samples were separated via $\mathrm{C}_{18}$ column (Phenomenex Prodigy ODS3, 5- $\mu \mathrm{m}$ particle size, Torrence, CA). Ergot alkaloids were quantified by comparing peak areas to a standard curve of dihydroergotamine and normalizing to number of conidia extracted. Conidia in each extract were counted with the aid of a hemocytometer.

The uncharacterized chemical detected via HPLC eluting from the column at 55.9 minutes was explored with the aid of a Thermo Fisher LCQ DecaXP LC/MS. Six replicates of $A$. fumigatus Af293 wild type, eas $M$ knockout, and eas $M$ complemented were grown on malt agar plates for two weeks. Spores were suspended in $5 \mathrm{~mL}$ HPLC grade methanol and scraped from the agar plates with a spreader. Spores were allowed to spin on a carousel for three hours and then were centrifuged for ten minutes. Each sample yielded slightly over one $\mathrm{mL}$ of extract which was subsequently concentrated to $100 \mu \mathrm{L}$ in a vacuum centrifuge. Of the resulting concentrate, $10 \mu \mathrm{L}$ was then loaded to be analyzed by LC/MS. LC/MS analyses were conducted as described by Ryan et al. (2013). 


\subsection{Statistical analysis}

Alkaloid concentrations were analyzed statistically with JMP software (SAS, Cary, NC) A Brown-Forsythe test was used to determine equality of group variances with $\mathrm{F}>0.05$ level. With equality of variances determined, an ANOVA was then used to compare quantities of each alkaloid among strains, with $\mathrm{p}<0.05$ indicating that chemical concentration varied by strain. This indication allowed for the subsequent Turkey-Kramer HSD test, to compare pairs to find significantly different means. Results reported in Table 2 display mean alkaloid concentrations \pm standard error. Letters A, B, or AB within each column indicate the results of the Tukey-Kramer test, with same letters showing no difference between strains and different letters indicating a statistically significant difference

\section{Results}

To test the hypothesis that eas $M$ is required for oxidation of festuclavine, eas $M$ was knocked out by replacing a large part of its coding sequences with a hygromycin resistance gene (Figure 6A). A complementation procedure was then carried out to restore the chemical profile and confirm eas $M$ function.

\section{1 easM knockout}

The native copy of eas $M$ in $A$. fumigatus was successfully knocked out through homologous recombination with the inactivated eas $M-\mathrm{HYG}$ construct. Of the 54 transformant samples tested by HPLC, 12 showed an accumulation of festuclavine (the intermediate preceding the hypothesized EasM step) and loss of fumigaclavine C, the pathway end product (Figure 7). An unknown chemical, eluting from the column at 55.9 minutes, also appeared to be affected by 
the eas $M$ knock out (Figure 7). Its identity was investigated via liquid chromatography mass spectrometry (LC/MS) in which a molecular ion of $m / z 309.3$ was detected (Figure 8 ). This molecular ion is consistent with the $[\mathrm{M}+\mathrm{H}]^{+}$for prenylated festuclavine, an alkaloid previously detected in A. fumigatus and named 9-deacetoxyfumigaclavine $\mathrm{C}$ by Ge et al. (2009).

Three types of PCR assays showed that the easM-HYG knockout construct was, in fact, integrated at the eas $M$ locus. The first assay was primed with oligonucleotides flanking the intended site of integration (Fig. 6B). The fragment obtained from the eas $M$ knockout (KO) was larger than that obtained from the wild type (WT) A. fumigatus due to the replacement of a portion of the eas $M$ coding sequences with the hygromycin resistance genes (Figure 6A). The second (5' screen) and third (3' screen) assays were each conducted with one primer that flanked the 5' or 3' end of eas $M$, respectively, and a second primer that annealed to the hygromycinresistance gene (Figure 6A). The samples showed predicted size bands for each screen (Figure 6). This indicated that the wild type sample contained a functional copy of eas $M$ as expected, the knockout strain contained the eas $M-\mathrm{HYG}$ construct which was integrated during fungal transformation, and that the complemented strain contained both the eas $M$ and eas $M$-HYG fragments.

\section{2 eas $M$ complementation}

To demonstrate that phenotype of the eas $M$ knockout was due to the integration at eas $M$ and not any ectopic events, the eas $M$ knockout strain was complemented in trans with the wildtype allele of eas $M$. Complementation resulted in restoration of the wild-type alkaloid profile in a qualitative sense (Figure 7). Integration of the complementing eas $M$ fragment was confirmed via PCR (Figure 6B). The presence of a fragment of wild-type length in the assay primed with 
eas $M$ forward and eas $M$ reverse primers indicated the incorporation of the wild-type allele, whereas the presence of the longer fragment (typical of the easM knockout strain) indicated that the complementing allele had integrated ectopically. Thus the primers amplified the eas $M$ wildtype copy as well as the easM-HYG construct at the previously knocked out eas $M$ locus, resulting in fragments of $3 \mathrm{~kb}$ and $3.4 \mathrm{~kb}$, respectively. Ectopic integration of the complementing allele also was supported by the observation of fragments typical of the knockout locus in the 5 , flank and 3' flank PCR assays (Figure 6B).

\subsection{Quantitative analysis of ergot alkaloids}

Table 2 displays comparisons of mean alkaloid accumulation in each strain \pm standard error. Festuclavine accumulated to significantly higher concentrations in the A. fumigatus easM knockout than in the A. fumigatus wild type and easM complemented strains $(\mathrm{P}<0.05)$.

Fumigaclavine B production could be detected in A. fumigatus wild type and complemented strains but was eliminated by the eas $M$ knock out. Quantities detected in wild type and complemented strains do not differ significantly.

Fumigaclavine $\mathrm{C}$ was detected in the wild type and complemented strains but was lacking in the eas $M$ knockout strain. The increase in concentration in the complemented strain suggests a difference in regulation of the ectopically integrated gene.

The unknown chemical, hypothesized to be prenylated festuclavine (Figure 8) was detected in all three strains. Knock out of eas $M$ resulted in a significant increase in concentration of this compound. The complemented strain produced an intermediate amount of chemical, thus not differing significantly from either wild type or from the knockout strain. 


\section{Discussion}

\subsection{Role of EasM in the ergot alkaloid pathway}

The pathway leading to the biosynthesis of lysergic acid is well established, but the pathway to dihydrolysergic acid-derived ergot alkaloids has not been elucidated. The objective of this study was to genetically manipulate steps in the ergot alkaloid pathway to prepare a strain of A. fumigatus suitable for future investigations of the biosynthesis of dihydrolysergic acid derived ergot alkaloids. Before exploring the dihydroergot alkaloid origin hypothesis, a festuclavine accumulator needed to be obtained. This was done by knocking out the relevant gene eas $M$ in the $A$. fumigatus ergot alkaloid pathway. Figure 4 illustrates the ergot alkaloid synthesis pathway in A. fumigatus. Genes responsible for the production of enzymes advancing each step of the pathway are indicated. However, the gene responsible for oxidizing festuclavine into fumigaclavine B had not been characterized. Dr. Panaccione and I hypothesized that the enzyme named EasM is responsible for oxidizing festuclavine to produce fumigaclavine B, which is then converted into downstream derivatives (Figure 4) based on its capacity to encode a P450 monooxygenase and its presence in the ergot alkaloid synthesis gene clusters of fumigaclavine producers. PCR and HPLC results along with the statistical analysis of this investigation described in the results section provide strong evidence that eas $M$ was successfully knocked out and complemented. These results indicate that the enzyme encoded by eas $M$ is necessary for oxidation of festuclavine into fumigaclavine B.

\subsection{Differences in alkaloid quantity among wild type, knockout, and complemented strains}

Table 2 shows a clear increase in the concentration of festuclavine and the putative prenylated festuclavine in the knockout mutant, this occurs because in the absence of EasM the 
pathway cannot advance and the fungus accumulates festuclavine (Figure 4) a portion of which becomes prenylated by easL. Other data in Table 2 may raise questions regarding the difference in alkaloid quantities between the wild type and complemented strains of A. fumigatus. One reason for the difference with higher quantities of alkaloid (fumigaclavine $\mathrm{C}$ and unknown chemical) produced in the complemented strain, may be attributed to the fact that the integration of the wild type eas $M$ gene into the complemented strain could not be controlled with precision. During the transformation procedure relatively high concentrations of the wild type eas $M$ gene were introduced into the protoplast mixture to facilitate higher probability of complementation. Multiple copies of the wild type eas $M$ gene could have integrated into the genome. Additionally, the integration locus may have affected the quantity of chemical the complemented fungus was then able to produce, as some portions of the genome are more regulated than others. Thus the quantity of $e a s M$ genes and their integration loci may be the explanatory factors for the difference in alkaloid quantity between the wild type and complemented A. fumigatus strains.

The transformation approach was chosen because it provided a reasonable strategy for analysis of gene function. The knockout construct was prepared in a relatively quick cloning and transformation procedure using a selectable marker, followed by a similar procedure for complementation. Phenotypes associated with ectopic integration of genes along with selectable markers could be reasonably differentiated via PCR and HPLC making this strategy a justifiable means for observing gene function.

The uncharacterized compound that accumulated to higher concentrations in the eas $M$ knockout strain of A. fumigatus (Figure 7 and 8 ) is hypothesized to be prenylated festuclavine. Rationale for this prediction in that festuclavine accumulates in the eas $M$ knockout strain due to the absence of a functional eas $M$ gene, the accumulated festuclavine may be utilized by an 
enzyme called EasL. EasL is a prenyl transferase whose typical function is to prenylate fumigaclavine A converting it into fumigaclavine C (Unsöld and Li 2006; Robinson and Panaccione 2012). Because fumigaclavine A is missing, due to the eas $M$ knockout (Figures 4 and 6) EasL may accept other substrates, in this case festuclavine, prenylating it into the compound that eluted at 55.9 minutes (Figure 7) and had a molecular ion of 309.3 Da (Figure 8). The ability of EasL to prenylate a variety of ergot alkaloids has been observed in previous studies (Ge et al. 2009; Robinson and Panaccione 2014).

Fumigaclavines $\mathrm{A}$ and $\mathrm{B}$ are intermediates between festuclavine and fumigaclavine $\mathrm{C}$ (Figure 4). Fumigaclavine A was not detected (Figure 7 and Table 2) in any of the strains used in this study. Absence of fumigaclavine A may be explained via function of EasL which converts fumigaclavine A to fumigaclavine C (Unsöld and Li 2006; Robinson and Panaccione 2012). Small quantities of fumigaclavine B were detected because the enzyme EasN had not yet converted fumigaclavine B to fumigaclavine A.

\subsection{Additional studies and future directions}

A. fumigatus, a fumigaclavine producer, and lysergic acid producers like Claviceps spp. and Epichloë spp. differ in their allele for eas $A$ within the ergot alkaloid pathway (Coyle et al. 2010). Epichloë sp. Lp1 CloA typically accepts agroclavine as its substrate and turns it into lysergic acid through a series of oxidations and a double bond isomerization (Robinson and Panaccione 2014). Expressing Epichloë sp. Lp1 cloA in an A. fumigatus festuclavine accumulator may test whether Epichloë sp. Lp1 CloA is able to accept festuclavine and turn it into dihydrolysergic acid. Agroclavine and festuclavine differ in their structure only by the presence or absence of a double bond in the $\mathrm{D}$ ring (Figure 3). One hypothesis is that eas $A$ is the 
only significant difference between dihydrolysergic and lysergic acid producers, with downstream enzymes in dihydrolysergic acid producers performing the same functions they do in lysergic acid producers.

Preliminary exploration of expressing an Epichloë cloA gene from an easA A. fumigatus promotor did not yield desirable results. The purpose of expressing cloA in an A. fumigatus festuclavine-accumulating strain was to test whether Epichloë sp. Lp1 native CloA, which typically modifies agroclavine into lysergic acid, would operate similarly in an A. fumigatus festuclavine-accumulating strain to produce dihydrolysergic acid. The core of lysergic and dihydrolysergic acid only differs by the presence of a double bond (Figure 5); festuclavine also possesses this same core (lacking a double bond) which may allow it to serve as a suitable substitute for the agroclavine substrate. Thus, CloA should add on functional groups in the two subsequent steps to make dihydrolysergic acid instead of the typical lysergic acid product (Figure 3). Additionally, evidence exists for permissiveness in substrate recognition in downstream enzymes; for example, LPS2 (Figure 3) in Claviceps purpurea has the ability to accept dihydrolysergic acid as well as lysergic acid as substrates (Riederer et al. 1996). This provides additional evidence that the enzymes of lysergic acid producing fungi have the ability to accept dihydrolysergic acid precursors. Epichloë sp. Lp1 cloA was expressed by fusing its coding sequences to the A. fumigatus eas $A$ promoter and the construct was transformed into the $A$. fumigatus festuclavine-accumulating strain. Conversion of festuclavine to lysergic acid was investigated via HPLC. Of the nineteen resulting transformants, integration of DNA was evident via PCR, but HPLC results did not indicate production of dihydrolysergic acid derivatives. Additionally, a feeding study testing the transformants ability to use exogenously supplied agroclavine and elymoclavine as substrates indicated that CloA was not utilizing the substrates 
for conversion to downstream products. There are many reasons why the expression of $c l o A$ may not have worked: damaged DNA, integration of construct at a non-ideal site in the genome, lack of affinity for festuclavine (a dihydrolysergic acid precursor) as a suitable substrate, or perhaps a combination of native alleles (eas $A$ and $\operatorname{clo} A$ ) are needed to make festuclavine work as a substrate for conversion to lysergic derivatives. Several fungi including A. fumigatus and Epichloë sp. have easA genes but their alleles differ. This may mean that the eas $A$ and cloA alleles from Epichloë may need to be transformed into A. fumigatus, as opposed to utilizing cloA from Epichloë sp. Lp1 and letting A. fumigatus utilize its own easA. Additional attempts have been made in utilizing eas $A$ and $c l o A$ from the same fungus, namely Epichloë but transformants did not result in desirable HPLC profiles. I suggest that future studies attempt to express other alleles of eas $A$ and $c l o A$ in the $A$. fumigatus festuclavine accumulator in an attempt to convert festuclavine to dihydrolysergic acid.

\subsection{Conclusion}

The ergot alkaloid synthesis cluster gene easM encodes a P450 monooxygenase that is necessary for oxidizing festuclavine into fumigaclavine B. Elucidating eas $M$ function is important as it adds to our knowledge of the ergot alkaloid pathway. Because eas $M$ had been sequenced but not yet characterized, this project provides evidence for easM's function which had previously not been documented. Moreover, the engineering of a festuclavine accumulating A. fumigatus strain is instrumental in the next phase of research for the biosynthesis of dihydrolysergic acid. Dihydrolysergic acid derivatives are vasorelaxant and are the active compounds in drugs that help treat disorders like dementia, and Alzheimer's. Agricultural 
applications for dihydroergot alkaloid producers which may counteract natural lysergic acid producing grass endophytes also exist. 


\section{Literature Cited}

Andersen, A. R., Tfelt-Hansen, P., \& Lassen, N. A. (1987). The effect of ergotamine and dihydroergotamine on cerebral blood flow in man. Stroke, 18, 120-123.

Bacon, C. W., \& White, J. F. (2000). Physiological adaptations in the evolution of endophytism in the Clavicipitaceae. Microbial endophytes, Marcel Dekker Inc., New York, NY, 237261.

Belser-Ehrlich, S., Harper, A., Hussey, J., \& Hallock, R. (2013). Human and cattle ergotism since 1900 Symptoms, outbreaks, and regulations. Toxicology and industrial health, 29, 307-316.

Bracco, F., Battaglia, A., Chouza, C., Dupont, E., Gershanik, O., Masso, J. F. M., \& Montastruc, J. L. (2004). The long-acting dopamine receptor agonist cabergoline in early Parkinson'sdisease. CNS drugs, 18, 733-746.

Clay, K., \& Schardl, C. (2002). Evolutionary origins and ecological consequences of endophyte symbiosis with grasses. The American Naturalist, 160, S99-S127.

Code of Federal Regulations (CFR), Title 7, Section 810 - Agriculture, Official US Standards for Grain, Jan 1, 2001 (www.access.gpo.gov/nara/cfr/cfr-table-search.html). 
Coyle, C. M., \& Panaccione, D. G. (2005). An ergot alkaloid biosynthesis gene and clustered hypothetical genes from Aspergillus fumigatus. Applied and environmental microbiology, $71,3112-3118$.

Coyle, C. M., Kenaley, S. C., Rittenour, W. R., \& Panaccione, D. G. (2007). Association of ergot alkaloids with conidiation in Aspergillus fumigatus. Mycologia, 99, 804-811.

Coyle, C. M., Cheng, J. Z., O'Connor, S. E., \& Panaccione, D. G. (2010). An old yellow enzyme gene controls the branch point between Aspergillus fumigatus and Claviceps purpurea ergot alkaloid pathways. Applied and environmental microbiology, 76, 3898-3903.

Denning, D. W. (1998). Invasive aspergillosis. Clinical infectious diseases, 781-803.

Ge, H.M., Yu, Z.G., Zhang, J., Wu, J.H., Tan, R.X. (2009). Bioactive alkaloids from endophytic Aspergillus fumigatus. Journal of Natural Products, 72, 753-755.

Hoffman, Albert (1980). LSD: My problem child. McGraw-Hill, New York, NY

Hofmann, A., Ratsch, C., \& Schultes, R. (1992). Plants of the Gods: Their Sacred, Healing, and Hallucinogenic Powers. Healing Arts Press, Rochester, VT, 124-135.

Fioravanti, M., \& Flicker, L. (2001). Nicergoline for dementia and other age associated forms of cognitive impairment. Cochrane Database of Systematic Reviews, 4. 
Grünewald, Matthias. Isenheim Altarpiece. (1510-1515). Oil on wood. Unterlinden Museum, Colmar, France.

Merkel, S., Dib, B., Maul, R., Köppen, R.., Koch, M., \& Nehls, I. (2012). Degradation and epimerization of ergot alkaloids after baking and in vitro digestion. Analytical and bioanalytical chemistry, 404, 2489-2497.

Panaccione, D. G., \& Coyle, C. M. (2005). Abundant respirable ergot alkaloids from the common airborne fungus Aspergillus fumigatus. Applied and environmental microbiology, 71, 3106-3111.

Panaccione, D. G., Cipoletti, J. R., Sedlock, A. B., Blemings, K. P., Schardl, C. L., Machado, C., \& Seidel, G. E. (2006). Effects of ergot alkaloids on food preference and satiety in rabbits, as assessed with gene-knockout endophytes in perennial ryegrass (Lolium perenne). Journal of agricultural and food chemistry, 54, 4582-4587.

Panaccione, D.G. (2010). Ergot alkaloids. The Mycota, Vol. 10, Industrial Applications, $2^{\text {nd }}$ Edition, Hofrichter, M. (Ed.). Springer-Verlag, Berlin-Heidelburg, 195-214.

Panaccione, D. G., Coyle, C. M., Schardl, C. L., Cheng, J. Z., O'Connor, S. E. (2012). Early steps and branch point of ergot alkaloid pathways in fungi. In Epichloae, endophytes of cool season grasses: implications, utilization and biology. Proceedings of the 7th 
International Symposium on Fungal Endophytes of Grasses, Lexington, Kentucky, 28

June to 1 July 2010, 127-131. Samuel Roberts Noble Foundation. http://www.noble.org/

Reddy, D. S. (2013). The pathophysiological and pharmacological basis of current drug treatment of migraine headache. Expert Review of Clinical Pharmacology, 6, 271-288.

Riederer, B., Han, M., \& Keller, U. (1996). D-Lysergyl peptide synthetase from the ergot fungus Claviceps purpurea. Journal of Biological Chemistry, 271, 27524-27530.

Robinson, S. L., \& Panaccione, D. G. (2012). Chemotypic and genotypic diversity in the ergot alkaloid pathway of Aspergillus fumigatus. Mycologia, 104, 11-310.

Robinson, S. L., \& Panaccione, D. G. (2014). Heterologous expression of lysergic acid and novel ergot alkaloids in Aspergillus fumigatus. Applied and environmental microbiology, 80, 6465-6472.

Schardl, C. L., \& Phillips, T. D. (1997). Protective grass endophytes: where are they from and where are they going? Plant disease, 81, 430-438.

Schardl, C. L., Leuchtmann, A., \& Spiering, M. J. (2004). Symbioses of grasses with seedborne fungal endophytes. Annual review of plant biology, 55, 315-340. 
Schardl, C. L., Panaccione, D. G., \& Tudzynski, P. (2006). Ergot alkaloids-biology and molecular biology. Alkaloids, 63, 45-86.

Schardl, C. L., Young, C. A., Hesse, U., Amyotte, S. G., Andreeva, K., Calie, P. J., \& Tanaka, E. (2013). Plant-symbiotic fungi as chemical engineers: multi-genome analysis of the Clavicipitaceae reveals dynamics of alkaloid loci. PLoS genetics, 9, e1003323.

Schardl, C. L., Young, C. A., Pan, J., Florea, S., Takach, J. E., Panaccione, D. G., \& Leuchtmann, A. (2013). Currencies of mutualisms: sources of alkaloid genes in vertically transmitted Epichloë. Toxins, 5, 1064-1088.

Scudamore K. A., Livesey C. T. (1997). Occurrence and significance of mycotoxins in forage crops and silage: a review. Journal of science and food agriculture, 77, 1-17.

Tudzynski, P., Correia, T., \& Keller, U. (2001). Biotechnology and genetics of ergot alkaloids. Applied microbiology and biotechnology, 57, 593-605.

Unsöld, I. A., \& Li, S. M. (2005). Overproduction, purification and characterization of FgaPT2, a dimethylallyltryptophan synthase from Aspergillus fumigatus. Microbiology, 151, 14991505.

Via, M. A., Chandra, H., Araki, T., Potenza, M. V., \& Skamagas, M. (2010). Bromocriptine approved as the first medication to target dopamine activity to improve glycemic control 
in patients with type 2 diabetes. Diabetes, metabolic syndrome and obesity: targets and therapy, 3, 43- 48.

Vollenweider, F. X., Vollenweider-Scherpenhuyzen, M. F., Bäbler, A., Vogel, H., \& Hell, D. (1998). Psilocybin induces schizophrenia-like psychosis in humans via a serotonin-2 agonist action. Neuroreport, 9, 3897-3902.

Wallwey, C., \& Li, S. M. (2011). Ergot alkaloids: structure diversity, biosynthetic gene clusters and functional proof of biosynthetic genes. Natural product reports, 28, 496-510. 
Figures and Tables
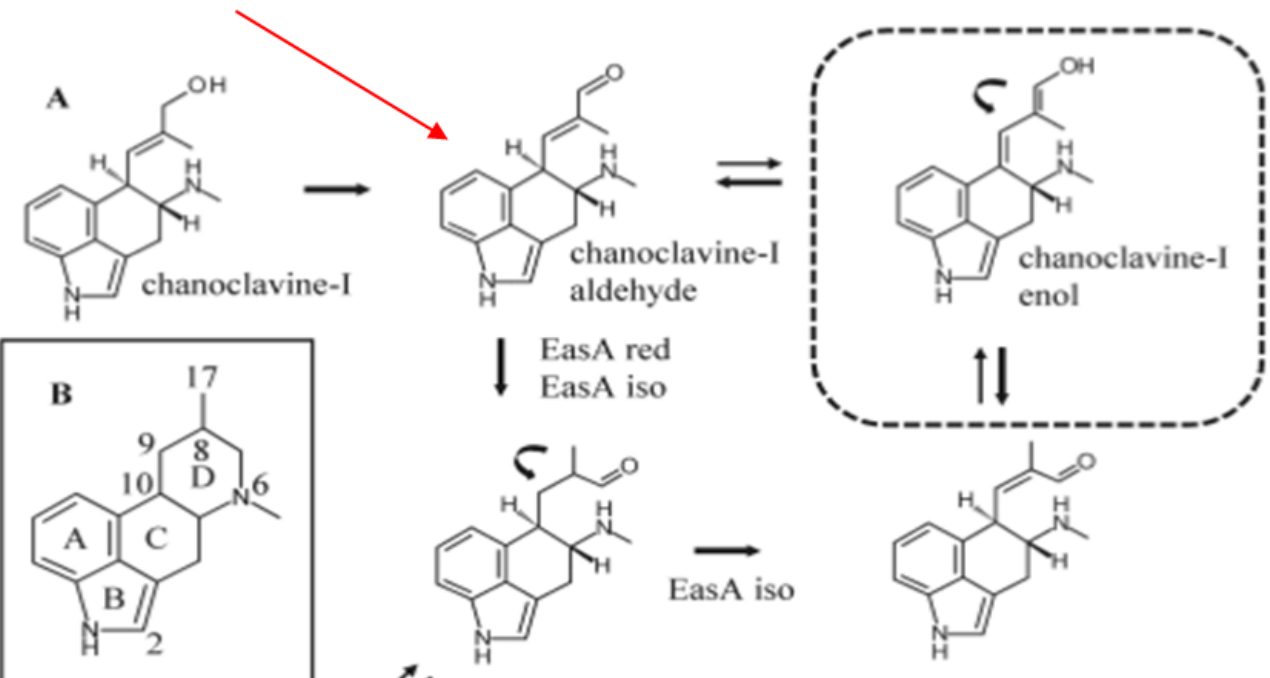

EasA red

EasA iso

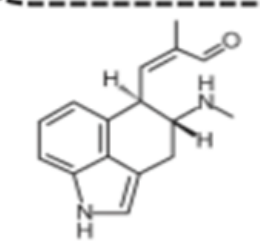<smiles>CC1C=[N+](C)[C@H]2Cc3c[nH]c4cccc(c34)C2C1</smiles><smiles>C=C[C@H]1Cc2c[nH]c3cccc(c23)C2C=C(C)[N+](C)=C[C@H]21</smiles><smiles>CC1C[C@H]2c3cccc4[nH]cc(c34)C[C@H]2N(C)C1</smiles>

festuclavine
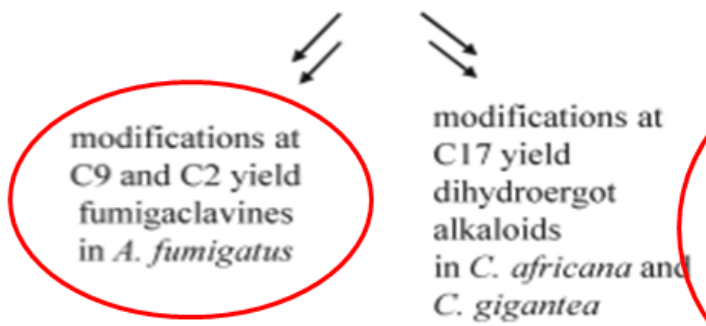

agroclavine
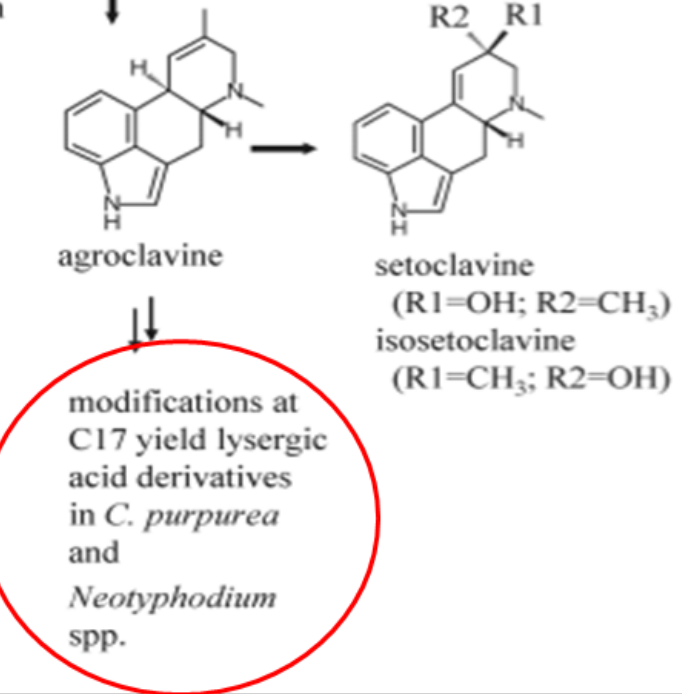

Figure 1: Divergence of the ergot alkaloid pathway at the chanoclavine-I aldehyde branch point. Note: Asexual Epichloë spp. were previously called Neotyphodium spp. The figure was modified from Panaccione et al. (2012). 
A. Aspergillus Fumigatus

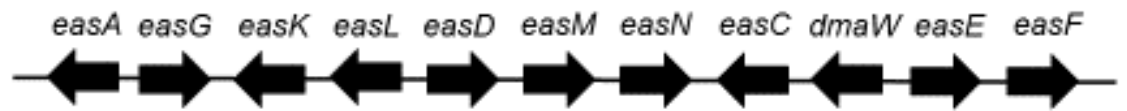

B. Epichloe sp. Lp1

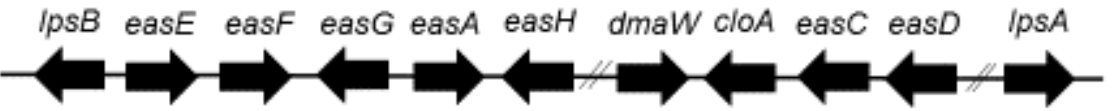

Figure 2: Ergot alkaloid synthesis gene cluster of $A$. fumigatus (A) redrawn from Coyle and Panaccione (2005). Epichlö̈ sp. Lp1 ergot alkaloid synthesis cluster (B) redrawn from Schardl et al. (2013). Compressed regions are designated with hash marks to allow for concise presentation. Arrows indicate direction of transcription. Functions have been assigned for all genes other than eas $M$ and easK. 

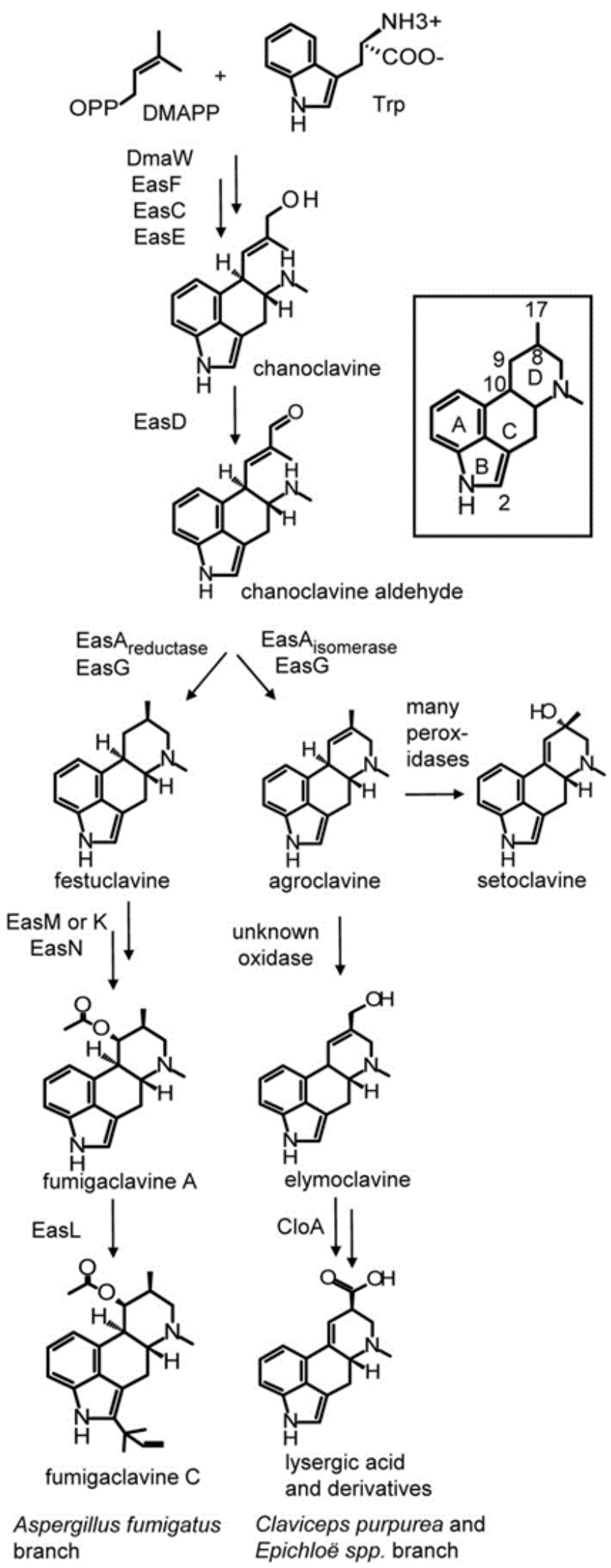

Figure 3: Ergot alkaloid pathway reproduced from Robinson \& Panaccione (2014). 


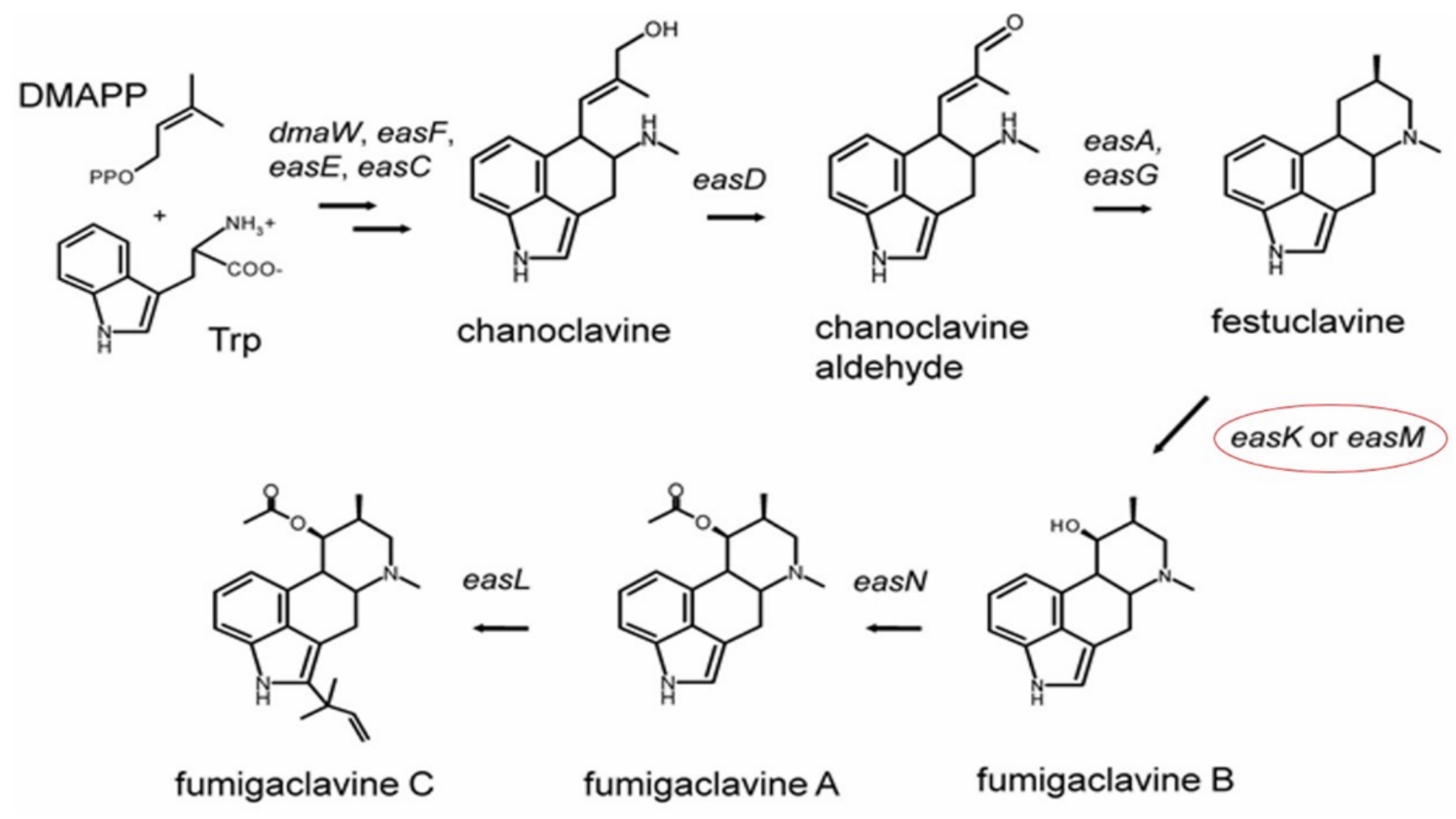

Figure 4: Ergot alkaloid pathway in A. fumigatus, showing genes (above corresponding arrows) responsible for each step (Panaccione 2010). 


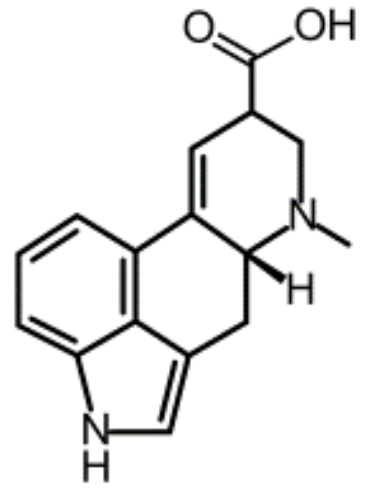

Lysergic Acid

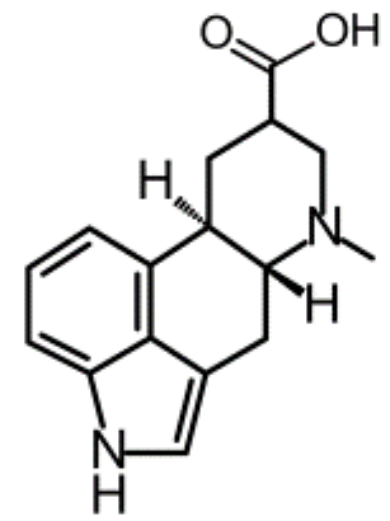

Dihydrolysergic acid

Figure 5: Lysergic acid and dihydrolysergic acid. The chemicals are the same except dihydrolysergic acid lacks a double bond in one of its rings. 
A.

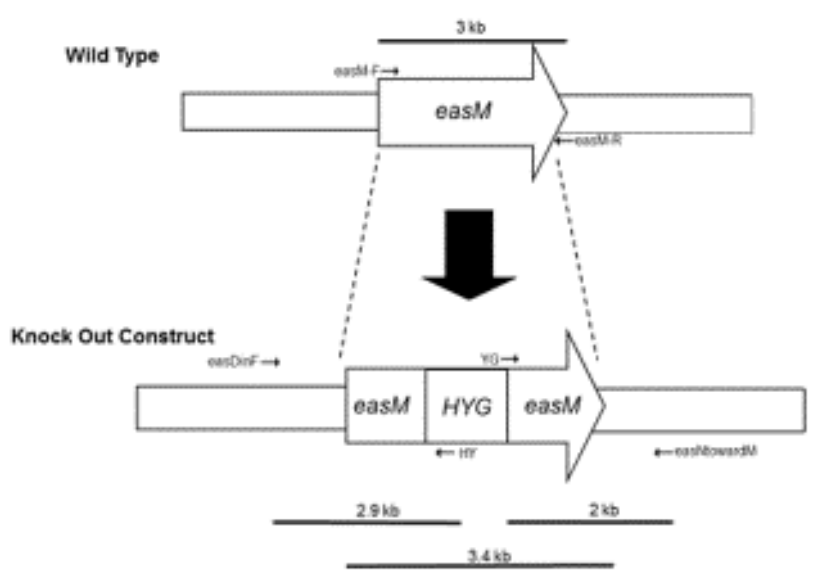

B.

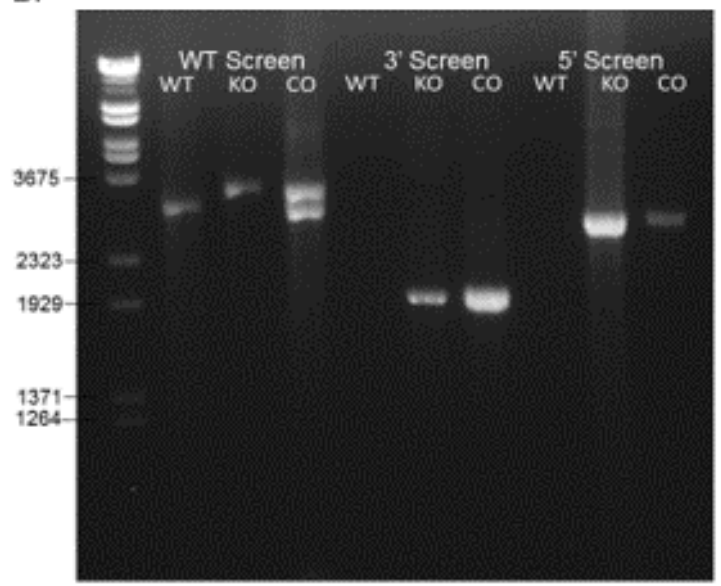

Figure 6: (A) Schematic representation of eas $M$ locus in A. fumigatus before and after gene knock out. (B) PCR products of $A$. fumigatus isolate Af293 wild type, eas $M$ knockout, and eas $M$ complemented strains. Wild type screen is checking for the eas $M$ fragment. Three prime screen was primed with primers that anneal beyond the 3' end of eas $M$ and the middle of the hygromycin resistance gene. Five prime screen was primed with oligonucleotides that anneal before the 5' end of eas $M$ and in the middle of the hygromycin resistance gene. 


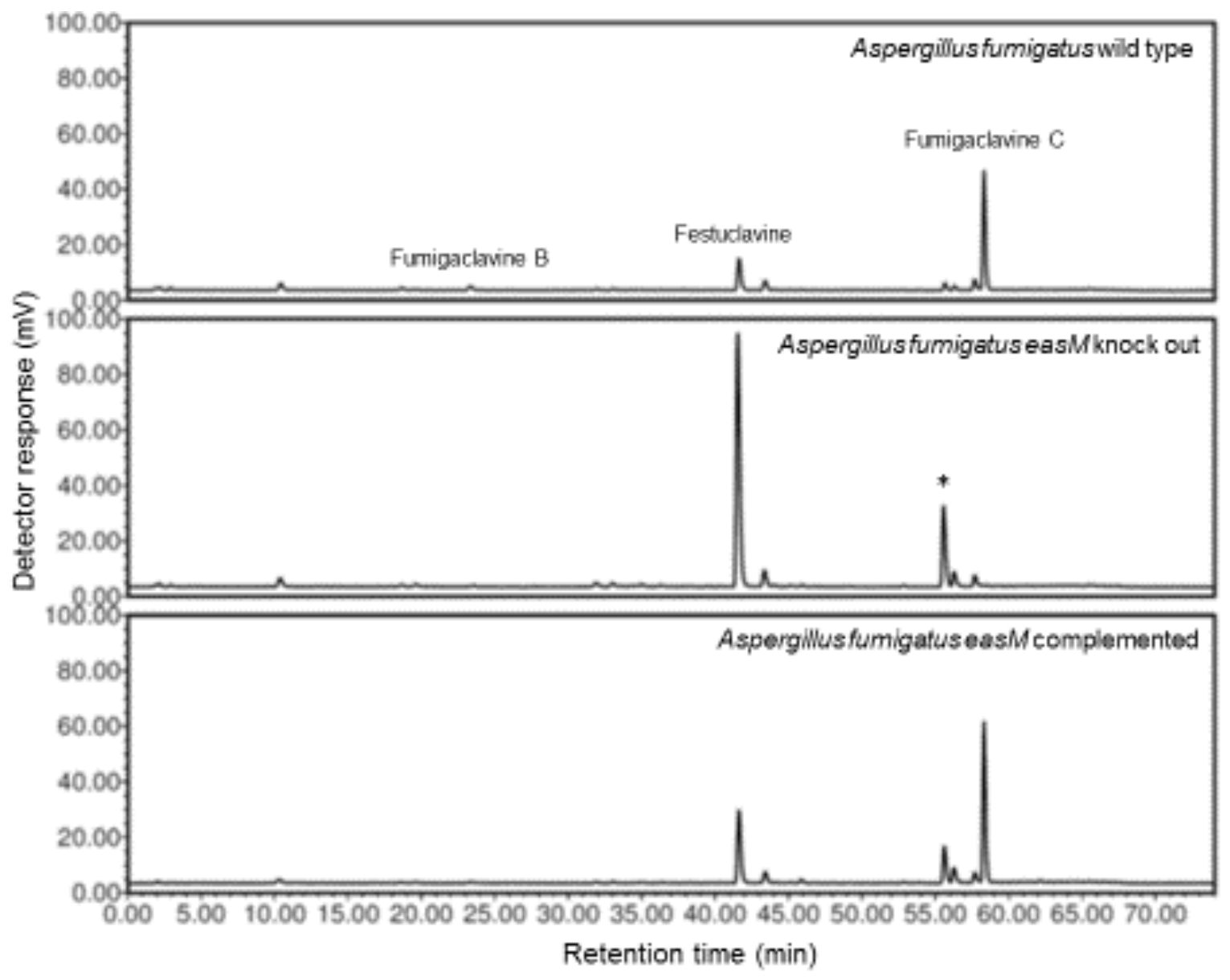

Figure 7: High performance liquid chromatography (HPLC) results illustrating ergot alkaloid accumulation in wild type, eas $M$ knock out, and complemented $A$. fumigatus strains. Data were collected with a fluorescence detector (excitation $272 \mathrm{~nm} /$ emission $372 \mathrm{~nm}$ ). Festuclavine eluted from the column at 41.9 minutes, fumigaclavine $\mathrm{C}$ eluted at 59 minutes, and a partially characterized compound (putative prenylated festuclavine; designated with an asterisk) eluted at 55.9 minutes. 


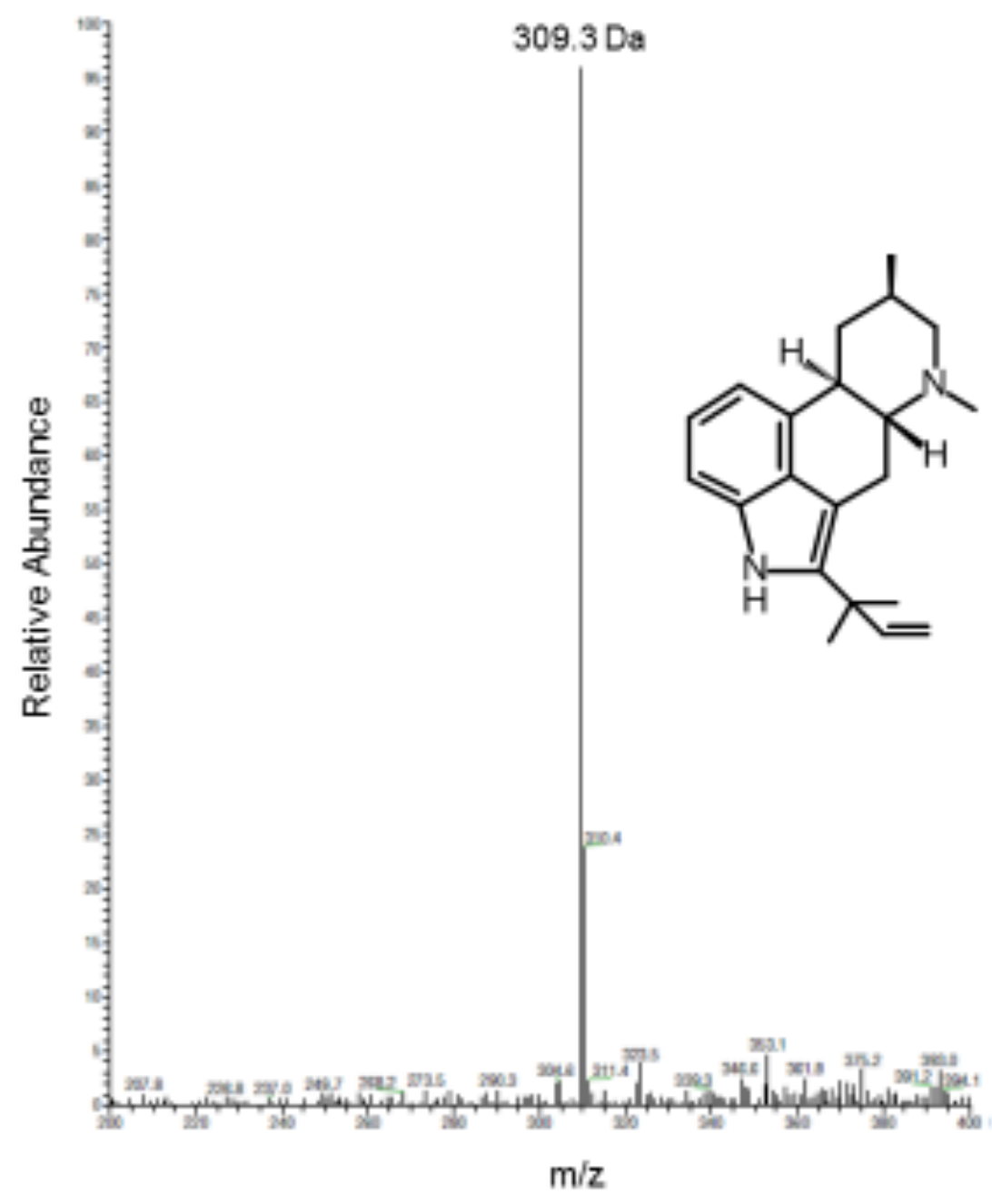

Figure 8: Mass spectrum and predicted structure of chemical accumulating to higher concentrations in the A. fumigatus eas $M$ knockout strain. 
Table 1 PCR primers used in this study and their products.

\begin{tabular}{|c|c|c|c|}
\hline $\begin{array}{c}\text { Primer } \\
\text { combination }\end{array}$ & Primer sequence $5^{\prime} \rightarrow 3^{\prime}$ & Product & $\begin{array}{l}\text { Amplicon } \\
\text { length (bp) }\end{array}$ \\
\hline $\begin{array}{c}\text { easMF + } \\
\text { easMR }\end{array}$ & $\begin{array}{l}\text { GCCATTCCTCCACTCTTCAG + } \\
\text { CCAAAGAACATTCCCAGCTC }\end{array}$ & easM & 3,067 \\
\hline $\begin{array}{c}\text { easNtowardM+ } \\
\text { YG }\end{array}$ & $\begin{array}{c}\text { GACTGCCAGTATCATCTACC + } \\
\text { CGTTGCAAGACCTGCCTGAA }\end{array}$ & $\begin{array}{l}\text { 3' flank of } \\
\text { integrated } \\
\text { ko construct }\end{array}$ & 2,057 \\
\hline $\begin{array}{l}\text { easDinF + } \\
\text { HY }\end{array}$ & $\begin{array}{c}\text { ACTGCGCTACAGTCGATGTAAC + } \\
\text { GGATGCCTCCGCTCGAAGTA }\end{array}$ & $\begin{array}{l}5 \text { flank of } \\
\text { integrated } \\
\text { ko construct }\end{array}$ & 2,916 \\
\hline
\end{tabular}


Table 2 Mean alkaloid concentration (mean amol/spore \pm standard error; $\mathrm{n}=6$ ) in A. fumigatus wild type, eas $M$ knockout, and eas $M$ complemented strains. Within each column, designation with different letters indicates significant difference $(\alpha=0.05)$ in a Tukey-Kramer HSD test (or one-way ANOVA, when alkaloid detected in two strains).

\begin{tabular}{lllll}
\hline Strain & Festuclavine & Fumigaclavine B & Fumigaclavine C & Unknown $^{\mathrm{a}}$ \\
\hline $\begin{array}{l}\text { A. fumigatus Af293 } \\
\text { wild type }\end{array}$ & $6.30 \pm 4.92 \mathrm{~A}$ & $0.58 \pm 0.09 \mathrm{~A}$ & $8.83 \pm 1.37 \mathrm{~A}$ & $1.19 \pm 0.90 \mathrm{~A}$ \\
$\begin{array}{l}\text { A. fumigatus Af293 } \\
\text { easM knockout }\end{array}$ & $33.59 \pm 9.19 \mathrm{~B}$ & n.d. $^{\mathrm{b}}$ & n.d. & $8.39 \pm 3.14 \mathrm{~B}$ \\
$\begin{array}{l}\text { A. fumigatus Af293 } \\
\text { easM complemented }\end{array}$ & $13.91 \pm 6.38 \mathrm{~A}$ & $0.35 \pm 0.05 \mathrm{~A}$ & $23.12 \pm 3.90 \mathrm{~B}$ & $4.64 \pm 3.72 \mathrm{AB}$ \\
\hline
\end{tabular}

${ }^{a}$ putative prenylated festuclavine

${ }^{b}$ not detected; limit of detection $=0.01 \mathrm{amol} / \mathrm{spore}$. 